\title{
Study on the UWB Rader Synchronization Technology
}

\author{
Guilin Lu \\ Guangxi University of Technology, Liuzhou 545006, China \\ E-mail: lifishspirit@126.com \\ Shaohong Wan \\ Ari Force No.95275, Liuzhou 545005, China \\ E-mail: wshhg@163.com \\ Kaiqing $\mathrm{Xu}$ \\ Ari Force No.95275, Liuzhou 545005, China
}

\begin{abstract}
In the article, the characteristics, synchronization method, approaches and process were described in detail, and the hardware platform of the system was designed. Furthermore, the article further pointed out that the research of the synchronization technology should be considered with the algorithm of channel estimation together, and the method of orthogonal wavelet could effectively solve the problem of channel estimation error.
\end{abstract}

Keywords: Synchronization, Channel estimation, Orthogonal wavelet

\section{Introduction}

The synchronization in the UWB radar system means making the code signals arriving at the receiver and the local reference signals consistent for the code design position and code clock rate on time, and it includes the capturing process and the following process. Though the uncertainties exist in the code phase and carrier frequency, but the receiver should decode signals normally, i.e. the resolving power of the carrier center frequency must make the dissociated signals in the region of correlative filter frequency, and make the local carrier frequency always aim at the carrier frequency of input signals to ensure the synchronization of code clock frequency. There are many methods such as the code synchronization, frequency synchronization, pulse synchronization and frame synchronization, and the capturing and following need not only the synchronization algorithms with good robustness, but the combination of the MB (multi-band) project which uses the frequency hopping and orthogonal frequency division multiplexing (OFDM) on the chip logic structure with the DS-UWB project which stores original UWB pulse attributes.

\section{Brief introduction of UWB synchronization technology}

The ultra wideband signals are transmitted to the receiver after modulating, and under the diffusion time delay extension and extent decline, these signals have been seriously distorted, and the pulse width of UWB carrier free is very narrow, and its performance is very sensitive with the time jitter. The demodulator is different with the receiver with narrowband wave, and it must use the channel estimation or the UWB receiver including the self-correlative receiver with equal performance when receiving or detecting, so the key technologies in the UWB radar technology are the synchronization technology and the channel estimation technology.

The synchronization processing includes following three steps (B. Pemson, 2001).

Step 1. Coarse synchronizing. The code phase receiving the pseudo-random code must be adjusted to the local pseudo-random code sequence.

Step 2. Code following and fine synchronizing. The phase separation of the pseudo-random code and the local pseudo-random code should be reduced to zero.

Step 3. Carrier return and carrier following. The picking and following of carrier phase can be implemented with code capturing and code following at the same time.

The hardware platform of synchronization technology in the UWB radar system is seen in Figure 1.

The fist task that the synchronization technology solves is to exactly know the frequency uncertainty induced by the diffusion distance and transmitting time, but the synchronization technology in the traditional IR-UWB interference system, the spread frequency synchronization technology in the DS-UWB and the synchronization technology in 
TR-UWB all have large loss of receiving signal to noise ratio, which would influence the precision, and because of the limitation of the hardware resources, the existing algorithm designs all need large amounts of data which are acquired by the statistical method, and the constringency speed is slow. Therefore, considering the influences of the synchronization deviation parameters on the synchronization performance, we should first confirm the coarse time delay, frequency deviation synchronization and channel estimation under the special realization demand of random channel. Large numbers of literatures adopted the iterative ML algorithm to optimize the maximum likelihood estimation algorithm, and up to now, the synchronization technology theory based on the wavelet theory has been applied broad.

\subsection{Research of capturing process algorithm}

For any measurement based on the receiving code byte sequence, if the result could show obvious difference in the synchronization state and the non-synchronization state, we can use this measurement to identify the synchronization and the non-synchronization. According to the characteristics of several modules in the synchronization technology, consider the influences of timing delay deviation parameters on the performances of synchronization, obtain the pulse position by the dichotomy searching, and capture the signal frame by the baker slide circuit.

Because of the carrier free characteristic of UWB, in the capturing aiming at the spread frequency, when $\mathrm{N}$ frequencies, $f_{1}, f_{2}, \ldots \ldots f_{N}$, enter into the capturing circuit based on the matching filter, the outputs from various branches are adjusted on time after different time delays, and enter into the comparator, and the input of the comparator is the frequency amount from the channel summitor, and if the correlative peak output producing the sum exceeds the adaptive threshold, so the system will capture the frequency hopping design, and obtain the initial synchronization signals, or else, the system should continue to capture the signals.

The principle figure of capturing circuit is seen in Figure 2.

\subsection{Characteristics of synchronization modules}

The modules in the synchronization process include frame synchronization module, symbol synchronization module, frequency synchronization module and pulse synchronization module, and various modules supplement each other.

The mathematical model of synchronization clock is based on the time processing process observed by the clock output port, and the clock equipment is composed by the oscillator and the counter.

The input UWB pulse waveform is

$s_{i}(t)=\left[1-4 \pi\left[\frac{t}{t_{w}}\right]^{2}\right] \exp \left(-2 \pi\left[\frac{t}{t_{w}}\right]^{2}\right)$

When the wave passes the synchronization module, the initial synchronization index signals can be denoted by

$s_{i}(t)=A_{i} c_{i}\left(t-i T_{i}\right) \sin \left[w_{i}\left(t-i T_{i}\right)+\theta_{i}\right]+n_{i}\left(t-i T_{i}\right)$.

Where, $n_{i}(t)$ is the Gauss white noise, $\theta_{i}(t)$ is the carrier initial phase of the i'th frequency hopping signal and the phase between the synchronization module and the receiving signal, $w_{i}(t)$ is the intermediate frequency after frequency conversion, and $c_{i}(t)$ is the $\mathrm{M}$ sequence signal.

The format of one frame in the frame synchronization is

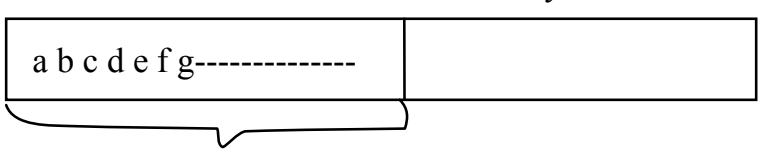

Frame header

The frame header of every frame is the pseudo-random sequence, and if the correlative function value between the transmitting sequence and the local sequence is detected, whether both phases are aligned can be judged, i.e. whether the capturing is successful can be judged, so the correct position of the frame header can be computed to directly demodulate the timing estimation and the frequency deviation estimation acquired before.

The synchronization characteristic of the code word and the code element is that if the spectrum character of the integral and differential signal and the spectrum character of the lead byte are consistent, and the power exceeds certain threshold, the synchronizations of both the code word and the code elements can be judged at the same time, and the information such as the frequency, phase and timing deviation needed by the digital transmission synchronization can be solved by taking the byte as the lead byte, which can ensure the coherence of the code phase and carrier frequency for receiver and transmitter.

We introduce the OFDM system into the UWB system, and the pilot frequency symbol can insert some known symbols and sequences on the frequency axis and the time axis simultaneously, and pick up the pilot frequency signal estimation channel transmission in the receiving port. 
The local sample signals are composed by the multi-path fading, time delay and other estimations, and the delay lock function and the implementation process are to calculate the local signals and receiving signals correlatively. Then the capturing enters into the second state, i.e. the coincidence detection state, and if the correlative function values of the local signal sequence and the transmission sequence are aligned and the threshold test is passed, the system enters into the following state, or else, the system will continue to process the pseudo-random code phase, and repeat the above process until capturing correct code element phase.

\subsection{United design theory of synchronization and channel estimation}

As the system enters into the following state from the capturing state, it can prevent the false alarms induced by the noise and the cheating signals, but the errors in the frequency deviation timing will influence the frequency synchronization technology, and the improved method is to comprehensively consider the synchronization and the channel estimation, and the channel adopts the cluster structure.

The improved method of the local sample signal is to adopt the channel estimation algorithm with high efficiency and multi-path extent channel parameters and the proper double-orthogonal wavelet mathematical model, which can constitute the local sample signals composed by the channel multi-path fading and time delay, and the slide correlative operation can realize the inner product of the signal and wavelet on the same scale, and avoid getting in the local minimum point process, and simplify the estimator circuit, and make the receiver capture the energy maximum of the signal, and effectively estimate many channel parameters such as the multi-path fading extent and time delay.

Because the false alarm probability and the capturing probability are a pair of ambivalent parameters, the estimation algorithm based on the maximum likelihood rules in the past was adopted to enhance the capturing performance of the receiver and realize the united design of the synchronization of the demand radar system and the channel estimation.

In the beginning of 1990s, the second order statistics was adopted to complete the blind equilibrium and blind identifying, but because the blind equilibrium of the second order matrix is sensitive to the estimation of the channel order number, the order number of the channel can not be exactly estimated in practice, so we introduce the higher-order-statistics (HOS) algorithm to solve this problem.

HOS contains the phase characteristics and the extent characteristics of the system or the signal, so it can be applied broad in the identification of the system, but its intolerant deficiency is the low constringency speed, and to acquire the estimation value, we need huge amounts of data samples, which makes the nonlinearity of the channel become very serious and lose the function.

To solve the problem of the bad channel receiving estimation errors aiming at ill-conditioned channel, the orthogonal wavelet channel estimation method was proposed in many literatures and acquired anticipated effects and objective.

\subsection{Application of wavelet theory in the synchronization technology}

To enhance the synchronization precision to the level of subnanosecond, UWB system adopts the orthogonal scaling function and the wavelet function waveform, and utilizes the $\mathrm{m}$ sequence theory to decompose the wavelet.

Schmidt orthogonal method is the method which solves a standard orthogonal basis from one basis a1, a2 ... in the $\mathrm{n}$ dimensional Euclidean space.

$b_{k+1}=a_{k+1}-\left(a_{k+1}, e_{1}\right) e_{1}-\cdots \cdots-\left(a_{k+1}, a_{k}\right) e_{k}$

For the non-stationary signal such as UWB, the basic wavelet function in the article is required to be oscillated and converged quickly. Based on the character that the wavelet or the wavelet network can approach any function or pulse response, and the orthogonal scaling transform can weaken the noise in the detected data, the whole synchronization circuit can work on Mcps.

The wavelet transform theory can be extensively applied in many domains such as signal processing, quantized filed theory and radar. The wavelet analysis overcomes the deficiencies in the traditional Fourier analysis, and it possesses good local characteristics in the time domain and frequency domain. The wavelet function is defined by the function cluster $\left\{\psi_{a b}\right\}$ from the base function $\psi(t)$ through flexing and levelly shifting.

$\psi_{a b}(t)=\frac{1}{\sqrt{|a|}} \psi\left(\frac{t-b}{a}\right)$

$a, b \in R, a \neq 0$

Because the wavelet function adopts the gradually refined time domain step length for the high frequency, so it can focalize any detail of the analyzed signal, and the important characteristics of the wavelet transform is that the wavelet transform possesses the gradual localization character, i.e. "varifocal".

The expression of the continuous wavelet is the inner product of the signal and the wavelet function, and the discretized wavelet generally adopts the binary discrete system, which can fulfill the orthogonal requirement, and the discretized 
continuous wavelet transform is generally used in the mallat algorithm of the filter group which can realize the reconstruction of the signal and largely reduce the computation. Figure 3 is the one dimensional signal reconstruction figure, and the discretized continuous wavelet transform adopts the mallat algorithm to recur the original waveform.

The multi-scale analysis to the signals in the article is to decompose the treating signals of the UWB radar system to different scales, and the former class signal is called as the smooth signal, and the latter disappearing signal is called as the detail signal, and the relations among signals on different scales are mainly completed by the wavelet transform.

The UWB radar signal input mode in this article is to evenly distribute the needed wavelet functions and scale functions designed by the UWB pulse waveform to multiple users (Zhu Lei Yu, 2004), utilize the fast algorithm of the orthogonal wavelet transform, i.e. the mallat algorithm, to quickly decompose and reconstruct the localization characteristics of the signals, realize the progressively taking process of the one-dimensional discreted wavelet transform of signal, separate the coarse weight and detail weight after discreted wavelet transform, transform the signals to the transmitting port of the UWB, implement wavelet transform to part of signals of the system and eliminate the detail information and transmit, reconstruct the original signals in the receiving port, implement discreted feedback to the difference between the original signals and the signals in the receiving port, and finally make the system achieve synchronization.

\section{Conclusions}

In this article, we researched the UWB radar synchronization technology in the implementation of all digital spread frequency, put forward the united design and research of the synchronization technology and the channel estimation by summarizing various characteristics of many UWB types such as DS-UWB, gave the hardware platform of the synchronization technology, the format of the frame, and the process of UWB from capturing to following, adopted the orthogonal wavelet channel estimation method to reduce the frequency deviation error. Base on the important character of the wavelet transform, the synchronization process of the UWB radar system is to process the wavelet coefficient decomposed by the threshold form, and reconstruct the signals to make the system to achieve the synchronization.

\section{References}

B. Pemson, D. E. DOd \& R. J. Bohon. (2001). A segmented matched filter for CDMA code synchronization in systems with Doppler frequency offset. Proceedings IEEE Glob . P_Abm, San Antonio, Texas. November 2001.

Zhu Lei Yu. (2004). Waveform Division Multiple Accesses Based on the Orthogonal Wavelet for Ultra-Wideband Impulse Radio Multiple-Access Communication. Journal of Beijing University of Posts and Telecommunications. No.27(4).

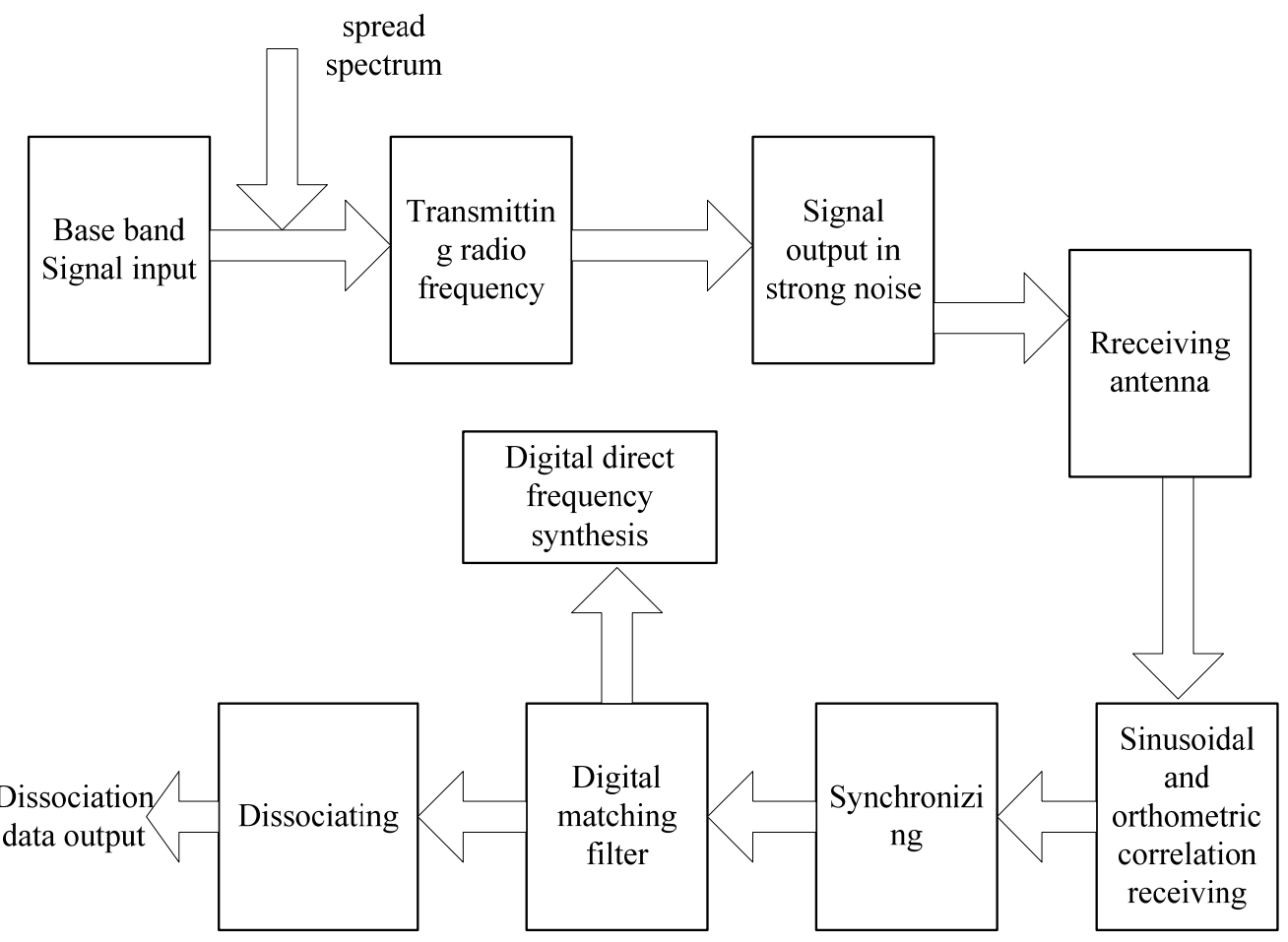

Figure 1. Hardware Platform of Synchronization Technology 


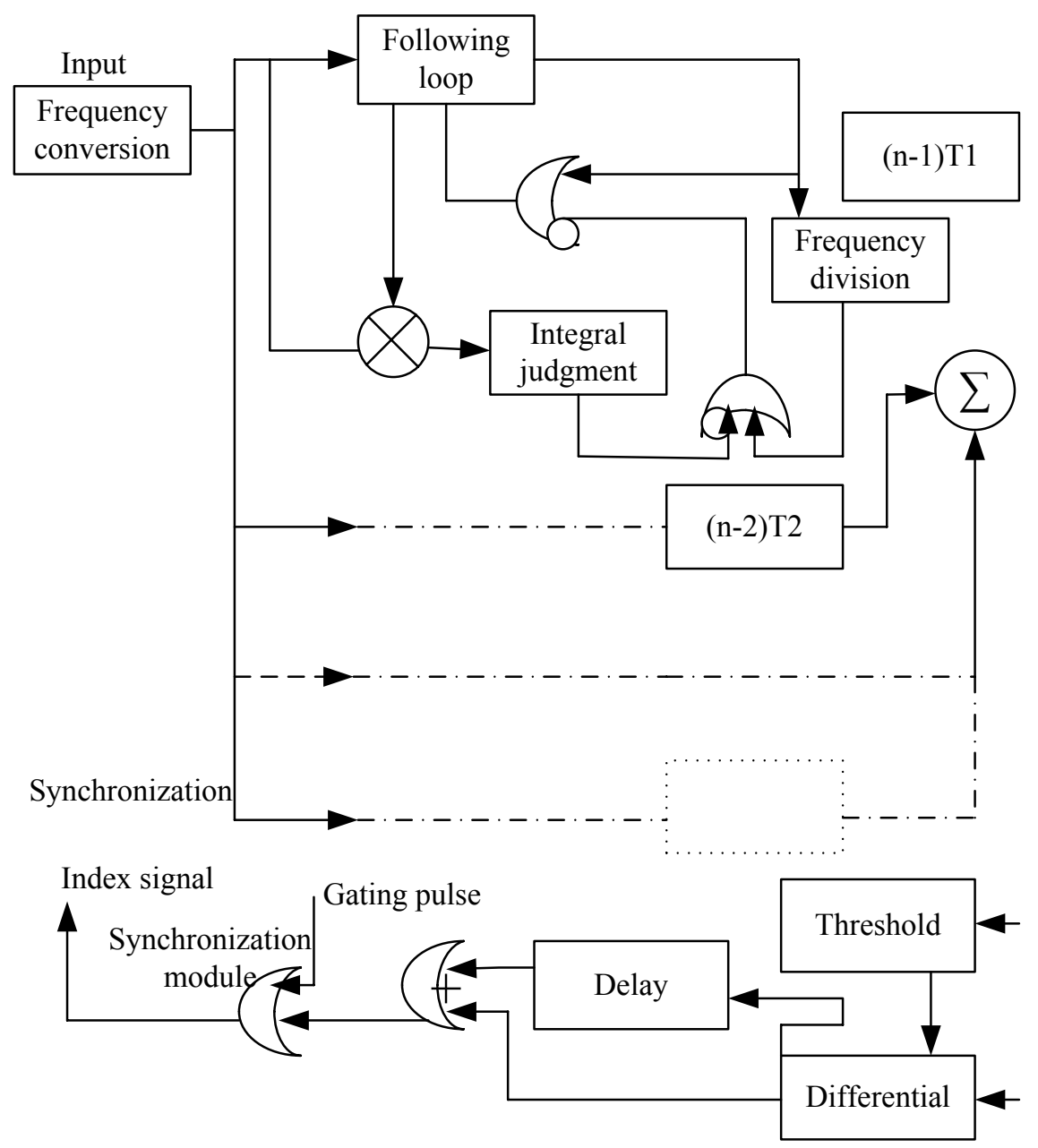

Figure 2. Capturing Circuit

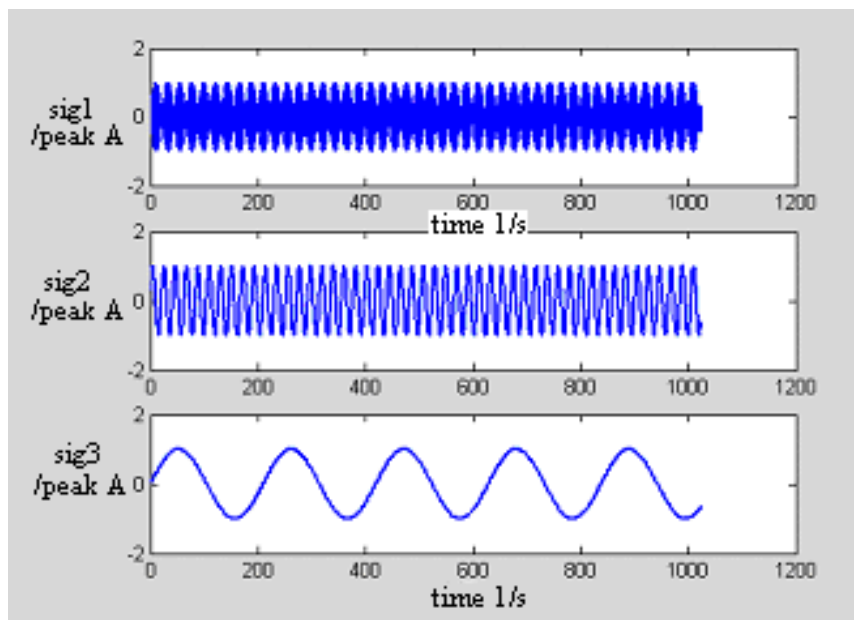

Figure 3. One-dimensional Wavelet Reconstruction 